\title{
Quantized Frame Expansions as Source-Channel Codes for Erasure Channels
}

\author{
Vivek K Goyal, Jelena Kovačević, and Martin Vetterli
}

\begin{abstract}
A new use for frame expansions is proposed. Previous investigations have revealed the robustness of frame expansions to additive noise and quantization. Here the robustness of quantized frame expansions to the loss ("erasure") of certain expansion coefficients is exploited in developing a source-channel code for erasure channels.
\end{abstract}

\section{INTRODUCTION}

Recently the problem of transmitting data over heterogenous networks has received considerable attention. A typical scenario might require data to move from a fiber link to a wireless link, which necessitates dropping packets to accommodate the lower capacity of the latter. If the network is able to provide preferential treatment to some packets, then the use of a multiresolution or layered source coding system is the obvious solution. But what if the network will not look inside packets and discriminate? Then packets will be dropped at random, and it is not clear how the source (or source-channel) coding should be designed. If packet retransmission is not an option (e.g., due to a delay constraint or lack of a feedback channel), one has to devise a way of getting meaningful information to the recipient despite the loss. The situation is similar if packets are lost due to transmission errors or congestion.

This problem is a generalization of the "multiple description" (MD) problem. In the MD problem, a source is described by two descriptions at rates $R_{1}$ and $R_{2}$. These two descriptions individually lead to reconstructions with distortions $D_{1}$ and $D_{2}$, respectively; and the two descriptions together yield a reconstruction with distortion $D_{0}$. The original problem, as posed by Gersho, Witsenhausen, Wolf, Wyner, Ziv and Ozarow in 1979, was to characterize the achievable quintuples $\left(R_{1}, R_{2}, D_{0}, D_{1}, D_{2}\right)$. The first design algorithm for practical MD coding was given by Vaishampayan [1]. For more background see [1] and the references therein.

Describing a source with $m$ descriptions such that any subset of the descriptions yields a useful reconstruction is a generalization of the MD problem. We propose the use of frame expansions from $\mathbb{C}^{n}$ to $\mathbb{C}^{m}(m>n)$ as a computationally simple approach to generalized MD coding.

\section{Frame Expansions with Erasures}

Let $F: \mathbb{C}^{n} \mapsto \mathbb{C}^{m}$ be the frame operator associated with the normalized tight frame $\Phi=\left\{\varphi_{k}\right\}_{k=1}^{m} \subset \mathbb{C}^{n},\left\|\varphi_{k}\right\|=1$ [2]. We propose to code source vectors $x \in \mathbb{C}^{n}$ by $Q(F(\cdot))$, where $Q$ represents scalar quantization followed by scalar entropy coding. The $m$ components of $\hat{y}=Q(F(x))$ are transmitted as separate descriptions of $x$. We analyze

V. Goyal and M. Vetterli are with the University of California, Berkeley. J. Kovačević is with Bell Laboratories, Lucent Technologies. M. Vetterli is also with École Polytechnique Fédérale de Lausanne. Comments and inquiries to v.goyal@ieee.org are welcome. here the distortion when $e$ of the descriptions are "erased."

Let $E$ denote the index set of the erasures, i.e., suppose $\left\{\left\langle x, \varphi_{k}\right\rangle\right\}_{k \in E}$ are erased. If $\Phi^{\prime}=\Phi \backslash\left\{\varphi_{k}\right\}_{k \in E}$ is a frame, the minimum MSE estimate $\hat{x}$ is obtained with the dual frame of $\Phi^{\prime}$; otherwise, $x$ can only be estimated to within a subspace and distributional knowledge is needed to get a good estimate. ${ }^{1}$ We model the quantizer as an additive white noise source, ${ }^{2}$ so $\eta=\hat{y}-F x$ has independent components and is independent of $x$ with $E\left|\eta_{k}\right|^{2}=\sigma^{2}$.

When there are no erasures, the error between the reconstructed signal $\hat{x}$ and source $x$ is due only to the quantization noise. The MSE in this case is $\mathrm{MSE}_{0}=$ $n^{2} \sigma^{2} / m$ [4]. Now suppose (renumbering, if necessary) $E=\{1,2, \ldots, e\}$ and let $F_{e}$ denote the frame operator associated with $\Phi^{\prime}$. The MSE can then be written as

$$
\begin{aligned}
\mathrm{MSE}_{e} & =\sigma^{2} \sum_{k=e+1}^{m}\left\|\left(F_{e}^{*} F_{e}\right)^{-1} \varphi_{k}\right\|^{2} \\
& =\left[1-\frac{e}{m}+\frac{n}{m^{2}} \sum_{i, j=1}^{e} B_{i j}^{(e)} C_{i j}^{(e)}\right] \mathrm{MSE}_{0},
\end{aligned}
$$

where $B^{(e)}$ and $C^{(e)}$ depend on the inner products between erased vectors. A simple special case is when the erased components are pairwise orthogonal. In this case, (1) reduces to $\mathrm{MSE}_{e}=(1+e /(m-n)) \mathrm{MSE}_{0}$.

\section{Remarks}

The coding method proposed here could be viewed as a signal-domain alternative to a (discrete) rate- $(n / m)$ block channel code. Though discussion here was limited to the general framework and the distortion with different numbers of erasures, preliminary calculations and simulations indicate potential for the proposed method. Compared to the use of a discrete channel code, the proposed method seems to give better performance at high rates and seems to be less sensitive to knowledge of the erasure probability. Minimum norm reconstructions can be computed in polynomial time for any $e$.

\section{REFERENCES}

[1] V. A. Vaishampayan, "Design of multiple description scalar quantizers," IEEE Trans. Inform. Th., vol. 39, no. 3, pp. 821834, May 1993.

[2] I. Daubechies, Ten Lectures on Wavelets, Society for Industrial and Applied Mathematics, Philadelphia, 1992.

[3] V. K Goyal and J. Kovačević, "Optimal multiple description transform coding of Gaussian vectors," in Proc. IEEE Data Compression Conf., Snowbird, Utah, Mar. 1998, pp. 388-397.

[4] V. K Goyal, M. Vetterli, and N. T. Thao, "Quantized overcomplete expansions in $\mathbb{R}^{N}$ : Analysis, synthesis, and algorithms," IEEE Trans. Inform. Th., vol. 44, no. 1, pp. 16-31, Jan. 1998.

\footnotetext{
${ }^{1}$ Extensions to where $\Phi^{\prime}$ is not a frame are suggested by [3].

${ }^{2}$ Better reconstructions are possible when the boundedness of the quantization error is exploited [4].
} 